EPJ Web of Conferences 81, 01011 (2014)

DOI: $10.1051 /$ epjconf/ 20148101011

(C) Owned by the authors, published by EDP Sciences, 2014

\title{
Signature of an $h_{1}$ state from $J / \psi \rightarrow \eta K^{* 0} \bar{K}^{* 0}$ and theoretical de- scription of the $Z_{c}(3900)$ and $Z_{c}(4020)$ as $D \bar{D}^{*}$ and $D^{*} \bar{D}^{*}$ molecular states
}

E. Oset ${ }^{1, a}$, Ju Jun Xie ${ }^{2}$, M. Albaladejo ${ }^{1}$, F. Aceti ${ }^{1}$, M.Bayar $^{3}$, J.M.Dias $^{1,4}$, A. Martinez Torres ${ }^{4}$, K. Khemchandani ${ }^{4}$, M. Nielsen ${ }^{4}$, F. Navarra ${ }^{4}$, X.L. Ren ${ }^{5}$, L.S. Geng ${ }^{5}$, Jie Meng ${ }^{5}$, and W. H. Liang ${ }^{6}$

${ }^{1}$ Departamento de Física Teórica and IFIC, Centro Mixto Universidad de Valencia-CSIC Institutos de Investigación de Paterna, Aptdo. 22085, 46071 Valencia, Spain

${ }^{2}$ Institute of Modern Physics, Chinese Academy of Sciences, Lanzhou 730000, China

${ }^{3}$ Department of Physics, Kocaeli University, 41380 Izmit, Turkey

${ }^{4}$ Instituto de Física, Universidade de São Paulo, C.P. 66318, 05389-970 São Paulo, SP, Brazil

${ }^{5}$ School of Physics and Nuclear Energy Engineering \& International Research Center for Nuclei and Particles in the Cosmos, Beihang University, Beijing 100191, China

${ }^{6}$ Department of Physics, Guangxi Normal University, Guilin, 541004, P. R. China

\begin{abstract}
In this talk we address two topics: The first one is an empirical explanation in terms of a new state $h_{1}$ of the peak in the $K^{* 0} \bar{K}^{* 0}$ invariant mass distribution close to threshold of this channel in the $J / \psi \rightarrow \eta K^{* 0} \bar{K}^{* 0}$ decay. The second one is a theoretical description of the isospin $I=1 Z_{c}(3900)$ and $Z_{c}(4020)$ states in terms of molecular states of $D \bar{D}^{*}+c c$ and $D^{*} \bar{D}^{*}$.
\end{abstract}

\section{Introduction}

In this talk we will address two topics: In the first topic we addreess the enhancement of the $K^{* 0} \bar{K}^{* 0}$ invariant mass distribution close to threshold in the $J / \psi \rightarrow \eta K^{* 0} \bar{K}^{* 0}$ decay and interprete it in terms of a new state $h_{1}$ which has been theoretically predicted as a dynamically generated state from the vector-vector interaction. In the second one we use and extension of the local hidden gauge approach for the interaction of mesons and provide a theoretical description of the $Z_{c}(3900)$ and $Z_{c}(4020)$ in terms of molecular states of $D \bar{D}^{*}$ and $D^{*} \bar{D}^{*}$.

\section{Experimental support for a new $h_{1}(1830)$ state}

The decay $J / \psi \rightarrow \eta K^{* 0} \bar{K}^{* 0}$ was measured by the BES Collaboration [1] searching for the $Y(2175)$ resonance, however, no clear enhancement in the $K^{* 0} \bar{K}^{* 0}$ mass distribution was found near 2.175 $\mathrm{GeV}$. Instead an enhancement in the $K^{* 0} \bar{K}^{* 0}$ mass distribution was found that passed unnoticed. In [2] this fact was used to claim support for an $h_{1}$ resonance that was predicted in [3] as dynamically generated from the interaction of vector mesons. The $h_{1}$ state has quantum numbers $0^{-}\left(1^{+-}\right)$, and,

\footnotetext{
a e-mail: oset@ific.uv.es
} 


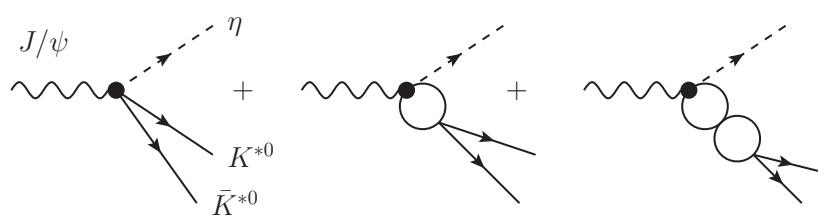

Figure 1. Diagrammatic representation of the $J / \psi \rightarrow \eta K^{* 0} \bar{K}^{* 0}$ decay.

since the $J / \psi$ and the $\eta$ mesons have quantum numbers $0^{-}\left(1^{--}\right)$and $0^{+}\left(0^{-+}\right)$, respectively, the decay $J / \psi \rightarrow \eta K^{* 0} \bar{K}^{* 0}$ constitutes the ideal reaction to look for an $h_{1}$ state, coupling to an $s$-wave $K^{*} \bar{K}^{*}$ pair. In Fig. 1 we show diagrammatically the process. The $K^{* 0} \bar{K}^{* 0}$ state is produced and the two $K^{*}$ interact. This interaction is the one that leads to the $h_{1}$ resonance in [3]. It is interesting to note that this state is very peculiar. Because of its negative C-parity, the $h_{1}$ state cannot couple to two vector mesons of the type $\rho, \omega, \phi$. Also because of its positive parity it cannot couple to two pseudoscalar mesons, which would have to be in $\mathrm{L}=1$ to match the spin of the $h_{1}$ and hence would have negative parity. The $K^{*} \bar{K}^{*}$ is then a unique state for both the construction and decay of this $h_{1}$ state.

The scattering matrix for the $K^{*} \bar{K}^{*}$ interaction is given in terms of a potential $V$ by

$$
t=v+v \widetilde{G} t=v(1+\widetilde{G} t)=(1-v \widetilde{G})^{-1} v=\left(v^{-1}-\widetilde{G}\right)^{-1},
$$

where $\widetilde{G}\left(M_{\text {inv }}^{2}\right)$ is the loop function for the $K^{*} \bar{K}^{*}$ pair. This function is divergent, and it can be regularized both with a cutoff or with dimensional regularization in terms of a subtraction constant. On the other hand the full amplitude for the process $J / \psi \rightarrow \eta K^{*} \bar{K}^{*}$ can be written, according to the diagrams in Fig. 1, as:

$$
t_{P}=V_{P}\left(1+\widetilde{G}\left(M_{\mathrm{inv}}^{2}\right) t\left(M_{\mathrm{inv}}^{2}\right)\right)=V_{P} \frac{t\left(M_{\mathrm{inv}}^{2}\right)}{v\left(M_{\mathrm{inv}}^{2}\right)},
$$

where the last equality follows from Eq. (1).

Our strategy to interpret the data of [1] is to use the potential $V$ derived in [3] and fit the data of [1] by changing the subtraction constant in the $\widetilde{G}$ function in dimensional regularization $a(\mu)$, where $\mu$ is a regularization scale, taken here as $\mu=1 \mathrm{GeV}$.

As seen in figure 2 a good agreement with the data is found for reasonable values of the parameter $a(\mu)$. The figure also shows the results with pure phase space normalized to the same area and we see that the disagreement with the data is quite obvious.

In order to see that with this parameter $a(\mu)$ one gets a resonance, in figure 3 we show the magnitude of $|t|^{2}$ and we see indeed that it has a resonant shape. We also perform an alternative fit to the data which could be carried on by someone not knowing the potential of [3]. In this case we can take a constant potential and fit it to the data. The results obtained are similar and the conclusion the same. Altogether we find from this analysis that there is a resonance $h_{1}$ with a mass $M_{h_{1}}=1830 \pm 20 \mathrm{MeV}$ and $\Gamma_{h_{1}}=110 \pm 10 \mathrm{MeV}$, respectively.

We think that these data offer enough evidence for this new state. In [4] we have made predictions for two more reactions $\eta_{c} \rightarrow \phi K^{*} \bar{K}^{*}$, or $\eta_{c}(2 S) \rightarrow \phi K^{*} \bar{K}^{*}$, that should help establish this resonance on firmer grounds. In Fig. 4 we show the predictions for $\eta_{c}(2 S) \rightarrow \phi K^{*} \bar{K}^{*}$ comparing the results with the $h_{1}$ resonance and pure phase space. The differences are striking and we encourage such a measurement to give further support to our claims based on the $J / \psi \rightarrow \eta K^{* 0} \bar{K}^{* 0}$ decay. 
MESON 2014 $-13^{\text {th }}$ International Workshop on Production, Properties and Interaction of Mesons

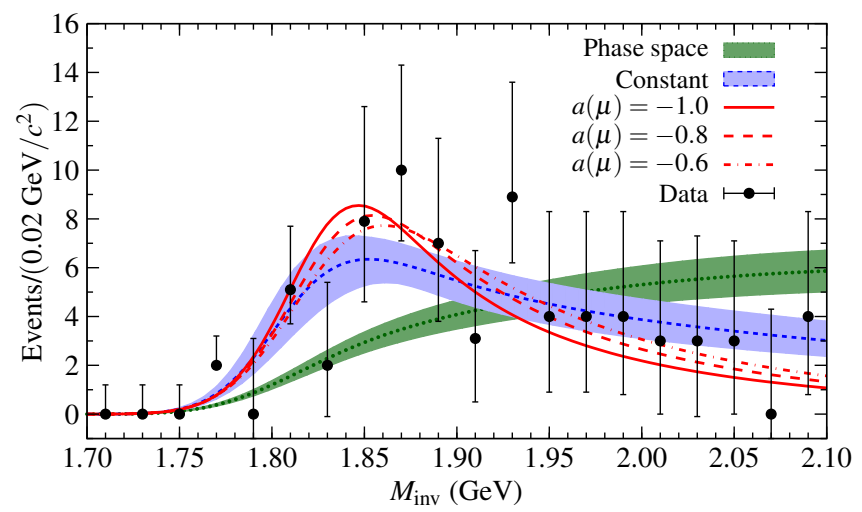

Figure 2. (Color online) The $K^{* 0} \bar{K}^{* 0}$ invariant mass spectrum of $J / \psi \rightarrow \eta K^{* 0} \bar{K}^{* 0}$ decay. The data points are taken from Ref. [1]. The different lines represent the output for the different approaches considered. The short-dashed line and the associated error band (light blue) represent the results of the constant potential. The (red) solid, long-dashed and dot-dashed lines represent the results for the vector exchange potential of [3] with $a(\mu)=-1.0$, -0.8 and -0.6 , respectively. Finally, the (dark green) dotted line, and the associated error band (green) is the prediction for phase space alone.

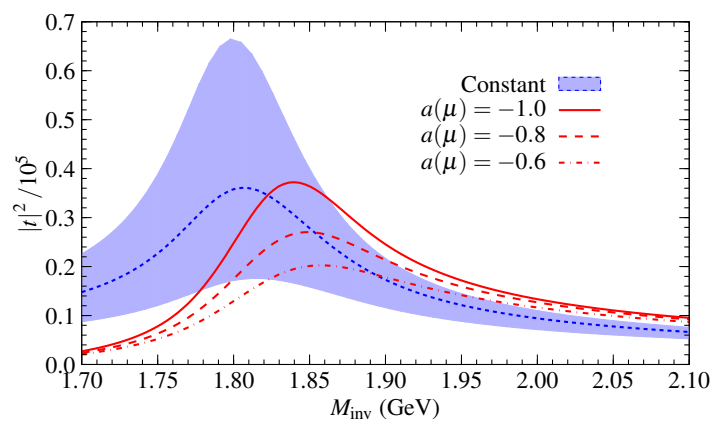

Figure 3. (Color online) The modulus squared of the amplitude, $|t|^{2}$, for $K^{*} \bar{K}^{*} \rightarrow K^{*} \bar{K}^{*}$, for the different approaches considered in this work. The notation of the lines as in Fig. 2.

In the charm sector there is also a related $h_{1}$ hidden charm state stemming from the vector vector interaction in [13] at $3945 \mathrm{MeV}$, which is also found in [5]. In [6] a reaction is also proposed to find this state, looking at the invariant mass distribution of $D^{*} \bar{D}^{*}$ in the $X(4660) \rightarrow \eta D^{*} \bar{D}^{*}$ decay.

\section{Theoretical description of the $Z_{c}(3900)$ and $Z_{c}(4020)$ as $D \bar{D}^{*}$ and $D^{*} \bar{D}^{*}$ molecular states}

One of the interesting recent surprises in hadronic physics has been the discovery of states that challenge the standard $q \bar{q}$ structure of the mesons. In this sense, charmonium states of isospin I=1 are of this type since $c \bar{c}$ has necessarily $\mathrm{I}=0$. One state called $Z_{c}(3900)$ has been reported by the BESIII collaboration [9] and reconfirmed in other experiments. Similarly, in [10] another $Z_{c}(4020)$ has been reported, also reconfirmed in other experiments. It is unclear whether this state has something to do 


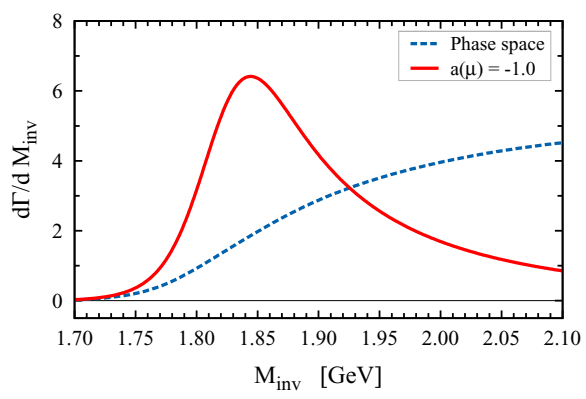

Figure 4. (Color online). The $K^{*+} K^{*-}$ invariant mass spectrum of $\eta_{c}(2 S) \rightarrow \phi K^{*} \bar{K}^{*}$ decay. The red solid line represents the results obtained with $a(\mu)=-1.0$, and the blue dashed line is the phase space.

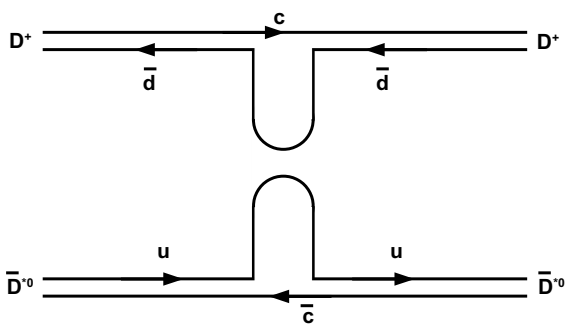

Figure 5. Feynman diagram depicting the OZI forbidden exchange of a light $q \bar{q}$ pair.

with the peak in the $D^{*} \bar{D}^{*}$ invariant mass distribution at $4025 \mathrm{MeV}$ in the $e^{+} e^{-} \rightarrow\left(D^{*} \bar{D}^{*}\right)^{ \pm} \pi^{\mp}$ reaction studied in [11]. Indeed, it was shown in [12] that this peak could be interpreted in terms of a resonance at much lower energy that was predicted in [13] from the $D^{*} \bar{D}^{*}$ interaction.

In $[7,8]$ we have looked into the possibility of creating states from the interaction of $D \bar{D}^{*}+c c$ and $D^{*} \bar{D}^{*}$ with isospin 1 . In order to study the interaction of pseudoscalar of vector mesons we use the local hidden gauge approach [14], which generalizes the chiral Lagrangians to include vector mesons. The approach produces the interaction by means of the exchange or vector mesons and several states can be produced with $\mathrm{I}=0$ in the charm sector [13], or in the bottom sector [15]. Yet, the $\mathrm{I}=1$ states are more difficult to obtain. The reason can be devised by looking at fig. 5. We can see that if we try to exchange a light $q \bar{q}$ state it is OZI forbidden since we would need to exchange a $d \bar{d}$ state for the upper line and the lower line demands a $u \bar{u}$ one. In view of this one has to exchange a heavy vector $c \bar{c}$, but one pays a penalty in this mechanism since the propagator of the heavy vector is much suppressed. This makes the interaction weaker and the possibilities of binding smaller. What we say about the exchange of a light vector can also be said about the exchange of pseudoscalar mesons and in $[7,8]$ it was shown that in the limit of equal masses for the pseudoscalar mesons, the contribution of the $\pi, \eta, \eta^{\prime}$ together vanished. In the case when different masses are taken into account then the cancellation is partial but the mechanism is also quite suppressed.

In view of this, in $[7,8]$ we also studied the exchange of two pions, either correlated to give the $f_{0}(500)$ or $\sigma$ meson through their interaction, or uncorrelated. They also were found to be relatively small. Yet, the strength of the heavy vector exchange, even if very much weakened, is enough to create some structure, pole or cusp, depending on the strength of the interaction which is governed by the cut 


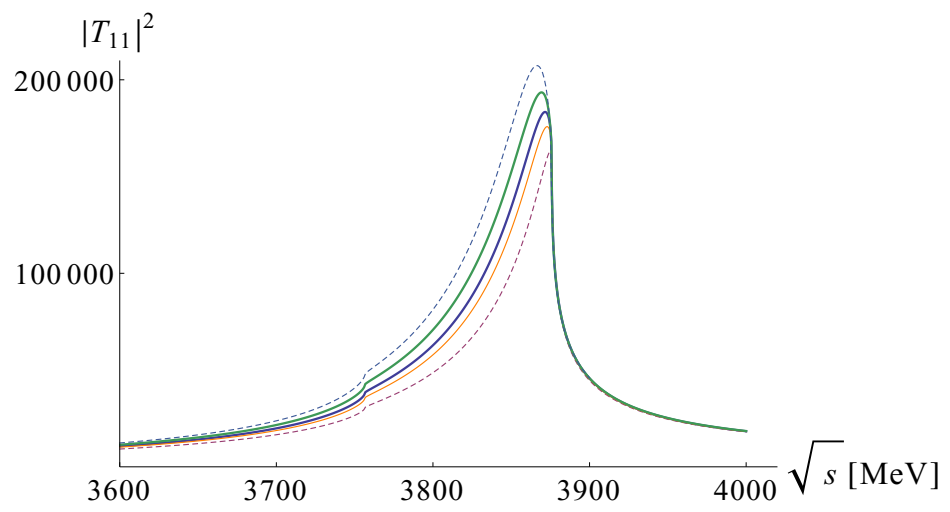

Figure 6. $|T|^{2}$ as a function of $\sqrt{s}$ for values of the cutoff $q_{\max }$ equal to $850,800,770,750$ and $700 \mathrm{MeV}$. Each line corresponds to a different cut off. The peak moves to the left as the cutoff increases.

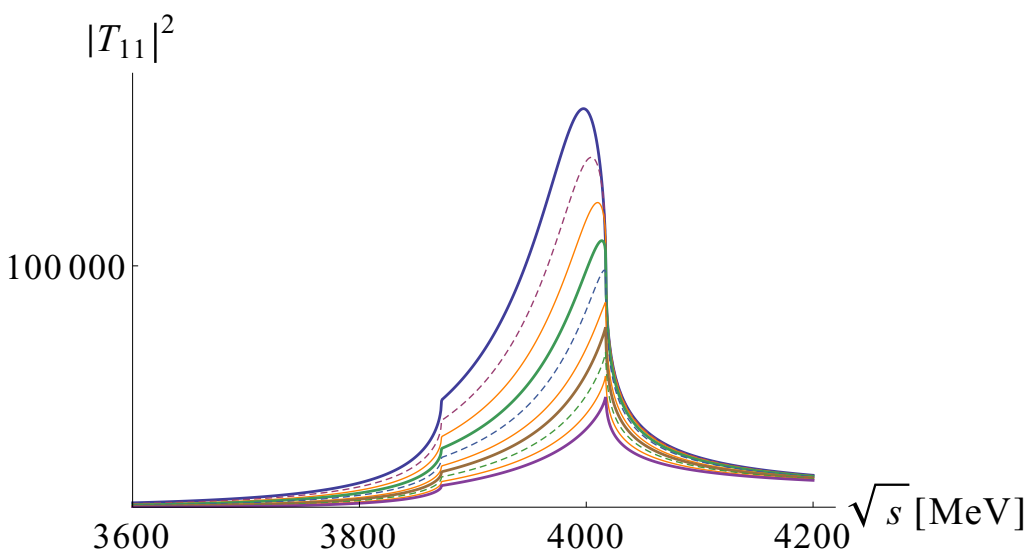

Figure 7. $\left|T_{11}\right|^{2}$ as a function of $\sqrt{s}$, for different values of the cutoff $q_{\max }$. Each line corresponds to a different cut off. From up down, $q_{\max }=960,900,850,800,750,700,650,600,550,500 \mathrm{MeV}$.

off of the $\mathrm{G}$ function. This we can see in Figs. 6 and 7 respectively for the case of the interaction of $D \bar{D}^{*}+c c$ and $D^{*} \bar{D}^{*}$. We can see in the figures that indeed the strength of $|T|^{2}$ accumulates around the thresholds of $D \bar{D}^{*}$ and $D^{*} \bar{D}^{*}$ respectively, and one could claim that a resonant state is obtained close to the threshold of these two channels, very close to what is observed in the experiment. In our case the width comes because the calculations are done in coupled channels and there are open channels, $\eta_{c} \rho$ and $\pi J / \psi$ in the case of the $D \bar{D}^{*}+c c$ states and $\rho J / \psi$ in the case of $D^{*} \bar{D}^{*}$ states. The width is about $50 \mathrm{MeV}$ for the case of the $D \bar{D}^{*}+c c$ state and about $100 \mathrm{MeV}$ for the case of the $D^{*} \bar{D}^{*}$ state, in qualitative agreement with experiment.

\section{Conclusion}

We have reported work on two topics. In the first one we showed how the data of the $J / \psi \rightarrow \eta K^{* 0} \bar{K}^{* 0}$ decay, by looking at the invariant mass distribution of the $K^{* 0} \bar{K}^{* 0}$, provided some evidence for a new 
$h_{1}(1830)$ state which is not catalogued in the PDG. We also suggested performing a measurement of two more reactions, $\eta_{c} \rightarrow \phi K^{*} \bar{K}^{*}$, or $\eta_{c}(2 S) \rightarrow \phi K^{*} \bar{K}^{*}$, that should help establish this resonance on firmer grounds. In the second part we explored the possibility of forming molecular states of $D \bar{D}^{*}+c c$ and $D^{*} \bar{D}^{*}$. We stressed the observation that from the point of view of meson exchange, the exchange of light vector mesons or pseudoscalar mesons is OZI forbidden. Then we explored the exchange of heavy vectors and two pion exchange, correlated and uncorrelated. We still found the exchange of vector mesons bigger than the two pion exchange, and enough to create some molecular structure very close to the threshold of the $D \bar{D}^{*}+c c$ and $D^{*} \bar{D}^{*}$ channels. We also included other pseudoscalar-vector and vector-vector states in a coupled channels formalism, which were open for the decay of the states found and they gave rise to a width in qualitative agreement with experiment, thus giving support for the $Z_{c}(3900)$ and $Z_{c}(4020)$ as weakly bound molecular states of $D \bar{D}^{*}+c c$ and $D^{*} \bar{D}^{*}$ respectively.

\section{Acknowledgements}

This work is partly supported by the Spanish Ministerio de Economia y Competitividad and European FEDER funds under the contract number FIS2011-28853-C02-01, and the Generalitat Valenciana in the program PrometeoII, 2014/068. J. M. Dias acknowledges the Brazilian Funding Agency FAPESP for support. We acknowledge the support of the European Community-Research Infrastructure Integrating Activity Study of Strongly Interacting Matter ( HadronPhysics3, Grant no. 283286) under the Seventh Framework Programme of EU and the National Natural Science Foundation of China under Grant No. 11165005 and Guangxi 201203 YB017.

\section{References}

[1] M. Ablikim et al. [BES Collaboration], Phys. Lett. B 685, 27 (2010)

[2] J. J. Xie, M. Albaladejo and E. Oset, Phys. Lett. B 728, 319 (2014)

[3] L. S. Geng and E. Oset, Phys. Rev. D 79, 074009 (2009)

[4] X. L. Ren, L. S. Geng, E. Oset and J. Meng, Eur. Phys. J. A 50, 133 (2014)

[5] J. Nieves and M. P. Valderrama, Phys. Rev. D 86, 056004 (2012)

[6] W. Liang, M. Albaladejo and E. Oset, Phys. Rev. D 88, 074027 (2013)

[7] F. Aceti, M. Bayar, E. Oset, A. M. Torres, K. P. Khemchandani, F. S. Navarra and M. Nielsen, Phys. Rev. D 90, 016003 (2014)

[8] F. Aceti, M. Bayar and E. Oset, Eur. Phys. J. A 50, 103 (2014)

[9] M. Ablikim et al. [BESIII Collaboration], Phys. Rev. Lett. 110, 252001 (2013)

[10] M. Ablikim et al. [BESIII Collaboration], Phys. Rev. Lett. 111, no. 24, 242001 (2013)

[11] M. Ablikim et al. [BESIII Collaboration], Phys. Rev. Lett. 112, 132001 (2014)

[12] A. Martinez Torres, K. P. Khemchandani, F. S. Navarra, M. Nielsen and E. Oset, Phys. Rev. D 89, 014025 (2014)

[13] R. Molina and E. Oset, Phys. Rev. D 80, 114013 (2009)

[14] M. Bando, T. Kugo and K. Yamawaki, Phys. Rept. 164, 217 (1988)

[15] A. Ozpineci, C. W. Xiao and E. Oset, Phys. Rev. D 88, 034018 (2013) 